\title{
Gradual Diffusive Capture: Slow Death by Many Mosquito Bites
}

\author{
S. Redner \\ Santa Fe Institute, 1399 Hyde Park Road, Santa Fe, New Mexico 87501, USA \\ Center for Polymer Studies and Department of Physics, Boston University, Boston, \\ MA 02215, USA
}

\section{O. Bénichou}

Laboratoire de Physique Théorique de la Matière Condensée (UMR CNRS 7600), Université Pierre et Marie Curie, 4 Place Jussieu, 75255 Paris Cedex France

\begin{abstract}
.
We study the dynamics of a single diffusing particle (a "man") with diffusivity $D_{M}$ that is attacked by another diffusing particle (a "mosquito") with fixed diffusivity $D_{m}$. Each time the mosquito meets and bites the man, the diffusivity of the man is reduced by a fixed amount, while the diffusivity of the mosquito is unchanged. The mosquito is also displaced by a small distance $\pm a$ with respect to the man after each encounter. The man is defined as dead when $D_{M}$ reaches zero. At the moment when the man dies, his probability distribution of displacements $x$ is given by a Cauchy form, which asymptotically decays as $x^{-2}$, while the distribution of times $t$ when the man dies asymptotically decays as $t^{-3 / 2}$, which has the same form as the one-dimensional first-passage probability.
\end{abstract}

PACS numbers: 02.50.Ey, 05.10.Gg, 05.40.Fb 


\section{Introduction}

One-dimensional diffusive capture processes have rich properties that have inspired much research in the applied probability [1 5] and statistical physics communities [6 10]. One example of this genre is the "lamb-lion" problem [11,12], in which a lamb diffuses on the infinite line in the presence of $N$ independently diffusing lions that initially are all to one side of the lamb. Whenever a lamb and lion meet, the lamb is killed. Asymptotically, the survival probability of the lamb due to these $N$ lions decays as $S_{N}(t) \sim t^{-\beta_{N}}$, where $\beta_{N}$ depends non-trivially on the number of lions [11,12] and secondarily on the diffusivities of the lamb and the lion, $D_{\ell}$ and $D_{L}$, respectively.

When $D_{\ell}=D_{L}$, the qualitative dependence of $\beta_{N}$ is understood, even though the exact value of $\beta_{N}$ is unknown for $N \geq 3$. For $N=1, \beta_{1}=\frac{1}{2}$, as this case can be mapped to the probability that a single random walker - corresponding to the lamb-lion separation - does not hit the origin by time $t$ [13 15]. For $N=2, \beta_{2}=\frac{3}{4}<2 \beta_{1}$ [11, 12]; that is, two lions are less effective in killing the lamb than what might be expected because of their ostensible independence. The resolution of this apparently contradictory behavior is that whenever the lamb moves, the lions move in unison with respect to the reference frame of the lamb. This induced correlation between the lions implies that the effective number of lions is less than 2. This three-body problem may be exactly solved by mapping it to the diffusion of a single effective particle in an absorbing twodimensional wedge of opening angle $\frac{2 \pi}{3}$ (and generally the opening angle is a function of $D_{\ell}$ and $D_{L}$ ) whose solution recovers $\beta_{2}=\frac{3}{4}$ [9, 11, 12, 15]; moreover, $\beta_{2}$ is readily computable for arbitrary $D_{\ell}$ and $D_{L}$ [15,16].

For $N \geq 3$, simulations give $\beta_{3} \approx 0.91342$ [17], $\beta_{4} \approx 1.03$, and $\beta_{10} \approx 1.4$ [1], but an analytical solution for $N \geq 3$ is not yet known. While the $N$-lion problem can be readily mapped onto the diffusion of an effective particle in $N+1$ dimensions that is restricted to an absorbing wedge region defined by $N$ constraint hyperplanes - the so-called Weyl chamber [25] - the solution to this simply-stated problem seems to be difficult to achieve. A simplification arises in the limit $N \rightarrow \infty$, however, where one can map the problem to the diffusion of an effective particle in the presence of an absorbing and approaching boundary whose position scales as $\sqrt{t}[18,23]$. This approach gives $\beta_{N} \simeq \frac{1}{4} \ln N$ for $N<\infty$ and $S_{\infty}(t) \simeq \exp \left(-\ln ^{2} t\right)$ for $N=\infty$ [11, 12,

Inspired by this lamb-lion system and also motivated by the unusual phenomenology of locally activated random walks [24], we introduce the "man-mosquitoes" model (Fig. 1), in which independent "mosquitoes", each with fixed diffusivity $D_{m}$, repeatedly bite the man that has an evolving diffusivity $D_{M}$. After each mosquito bite, the diffusivity of the man is reduced by a fixed amount $\Delta D$, while the diffusivity of each mosquito is unchanged. To avoid spurious singularities, the mosquito immediately moves equiprobably either a distance $+a$ or $-a$ from the man after each encounter. Thus after each mosquito bite, the encounter process begins anew, but with the diffusivity of the man reduced by $\Delta D$. For convenience, we define the initial diffusivity of the man $D_{M}$ to be an integer multiple of $\Delta D$, that is, $D_{M}=\mathcal{N}(\Delta D)$. Thus after being bitten 


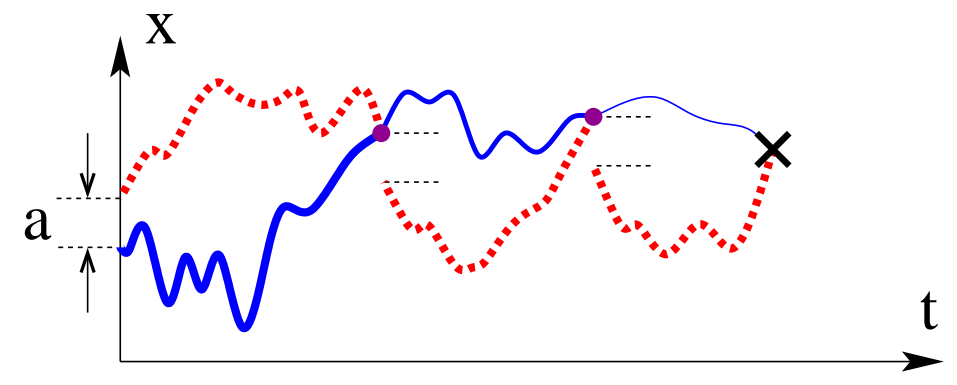

Figure 1. Space-time evolution of a single mosquito (dashed) and man (solid). After each encounter, the mosquito is displaced by $\pm a$ with respect to the man, while the man becomes progressively more sluggish and eventually comes to rest.

$\mathcal{N}=D_{M} / \Delta D$ times, the man is at rest, which we define as being "dead". Our goal is to determine the distribution of times and distances traveled after each encounter with the mosquitoes and the position and time elapsed when the man dies.

Here we treat the simplest, but still non-trivial, case of a single mosquito. Initially, the man is at $x_{M}=x_{0}$ and the mosquito is equiprobably at either $x_{m}=x_{0}+a$ or $x_{0}-a$, where $a$ can be viewed as the lattice spacing in a discrete random-walk formulation. The basic ingredient to understand how the man moves is determined by the statistical properties of the first encounter between the man and mosquito when they have arbitrary diffusivities and start a distance $a$ apart. The full problem involves convolving $\mathcal{N}$ of these first encounters. To solve for a single first encounter, we map the one-dimensional motions of the two particles onto the motion of a single effective particle in two dimensions, whose coordinates are the positions of the man and the mosquito, and then apply the image method to solve this effective problem (Sec. 22). In section 3, we apply these single-encounter results to determine the dynamics of multiple encounters. By this approach, we thereby determine the probability distributions of displacements and lifetimes of the man at the moment when he dies. We provide a brief discussion and outline several extensions of this work in Sec. 4.

\section{Single Man-Mosquito Encounter}

\subsection{Equal Diffusivities}

As a preliminary, we review the first-passage properties of an isotropically diffusing particle in two dimensions that is initially located at $(x, y)=(0, a)$, diffuses in the half space $y>0$, and is absorbed when it hits any point $x$ along the locus $y=0$ [13 15]. This absorption is characterized by: (i) the first-passage probability, namely, the probability that the particle first hits a point $(x, 0)$ on the absorbing line at time $t$, and (ii) the eventual hitting probability, the probability that particle is eventually absorbed at $(x, 0)$.

The probability density of the particle, $c(x, y, t)$, obeys the diffusion equation, $\partial_{t} c=D \nabla^{2} c$, subject to the absorbing boundary condition $c(x, 0, t)=0$ and the initial condition $c(x, y, t=0)=\delta(x) \delta(y-a)$. By the image method, the solution is a sum of a 
Gaussian centered at $(0, a)$ and an anti-Gaussian centered at the image point $(0,-a)$ :

$$
c(x, y, t)=\frac{1}{4 \pi D t}\left\{e^{-\left[x^{2}+(y-a)^{2}\right] / 4 D t}-e^{-\left[x^{2}+(y+a)^{2}\right] / 4 D t}\right\} .
$$

From this expression, the first-passage probability to the point $(x, y=0)$ is

$$
F(x, t)=\left.D \frac{\partial c}{\partial y}\right|_{y=0}=\frac{a}{4 \pi D t^{2}} e^{-\left(x^{2}+a^{2}\right) / 4 D t} .
$$

Notice that while the flux, $-D \frac{\partial c}{\partial y}$, is in the $-y$ direction, the first-passage probability is positive. Integrating this first-passage probability over all time gives the eventually hitting probability to a point $(x, 0)$ on the $x$-axis. Using the variable substitution $u=\left(x^{2}+a^{2}\right) / 4 D t$, this integration becomes elementary and the result is the Cauchy, or Lorenztian, distribution:

$$
E(x)=\int_{0}^{\infty} F(x, t) d t=\frac{1}{\pi} \frac{|a|}{x^{2}+a^{2}} .
$$

The absolute value sign makes this result valid for $a>0$ and $a<0$. Because of this power-law decay, the mean-square position of the particle when it hits the absorbing line is infinite. Parenthetically, the probability that the particle first hits any point on the $x$-axis at time $t$ is simply the classic one-dimensional first-passage probability

$$
f(t)=\int_{-\infty}^{\infty} \frac{|a|}{4 \pi D t^{2}} e^{-\left(x^{2}+a^{2}\right) / 4 D t} d x=\frac{|a|}{\sqrt{4 \pi D t^{3}}} e^{-a^{2} / 4 D t},
$$

for which the mean first-hitting time is divergent.

\subsection{General Diffusivities}

We now study the case of a man with diffusivity $D_{M}$ and mosquito with diffusivity $D_{m}$ that are initially at $x_{M}=x_{0}$ and $x_{m}=x_{0}+a$, respectively; later we will average over the cases where initially $x_{m}=x_{0}+a$ and $x_{m}=x_{0}-a$. To determine the probability that the man and mosquito first meet at position $x$ at time $t$ when starting from this initial state, we map this two-particle system to an effective single-particle problem in the two-dimensional coordinates $\left(x_{M}, x_{m}\right)$. The diffusion coefficient of the effective particle is anisotropic, since typically $D_{M} \neq D_{m}$. To simplify matters, we rescale the effective particle coordinates to $y_{M}=x_{M} / \sqrt{D_{M}}$ and $y_{m}=x_{m} / \sqrt{D_{m}}$ so that the effective particle diffuses isotropically with unit diffusivity. The condition that the mosquito meets the man, $x_{M}=x_{m}$, translates to $y_{M} \sqrt{D_{M}}=y_{m} \sqrt{D_{m}}$. Thus the effective particle starts at $\left[x_{0} / \sqrt{D_{M}},\left(x_{0}+a\right) / \sqrt{D_{m}}\right]$, moves with unit diffusivity, and eventually hits the line

$y_{M} \sqrt{D_{M}}=y_{m} \sqrt{D_{m}}$ that is inclined at an angle $\theta=\tan ^{-1}\left(\sqrt{D_{M} / D_{m}}\right)$ with respect to the horizontal (Fig. 2).

To analyze this problem, it is convenient to introduce the rotated coordinates $z_{M}, z_{m}$ defined by

$$
\begin{aligned}
& z_{M}=y_{M} \cos \theta+y_{m} \sin \theta=\frac{x_{M}}{\sqrt{D_{M}}} \cos \theta+\frac{x_{m}}{\sqrt{D_{m}}} \sin \theta, \\
& z_{m}=y_{m} \cos \theta-y_{M} \sin \theta=\frac{x_{m}}{\sqrt{D_{m}}} \cos \theta-\frac{x_{M}}{\sqrt{D_{M}}} \sin \theta,
\end{aligned}
$$




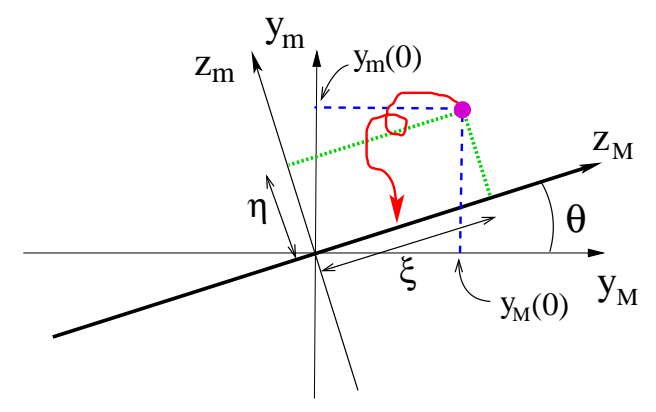

Figure 2. Mapping the diffusion of a man and mosquito on the line to diffusion in the half space $z_{m}>0$. A first-passage trajectory is shown.

because the mosquito meeting the man corresponds to the effective particle hitting a point on the locus $z_{m}=0$. In these rotated coordinates, the initial condition becomes

$$
\begin{aligned}
& z_{M}(0) \equiv \xi=\frac{x_{M}(0)}{\sqrt{D_{M}}} \cos \theta+\frac{\left(x_{M}(0)+a\right) \sin \theta}{\sqrt{D_{m}}}=\frac{x_{M}(0)}{\sqrt{D_{M}} \cos \theta}+\frac{a \sin \theta}{\sqrt{D_{m}}} \\
& z_{m}(0) \equiv \eta=\frac{\left(x_{M}(0)+a\right) \cos \theta}{\sqrt{D_{m}}}-\frac{x_{M}(0)}{\sqrt{D_{M}}} \sin \theta=\frac{a \cos \theta}{\sqrt{D_{m}}}
\end{aligned}
$$

Following Eq. (2), the probability density of the effective particle in the $z$-plane is again the sum of a Gaussian and an anti-Gaussian:

$$
c\left(z_{M}, z_{m}, t\right)=\frac{1}{4 \pi t}\left\{e^{-\left[\left(z_{M}-\xi\right)^{2}+\left(z_{m}-\eta\right)^{2}\right] / 4 t}-e^{-\left[\left(z_{M}-\xi\right)^{2}+\left(z_{m}+\eta\right)^{2}\right] / 4 t}\right\} .
$$

From this expression, the first-passage probability to a point $z_{M}$ on the $z_{m}=0$ axis is

$$
F\left(z_{M}, t\right)=\frac{|\eta|}{4 \pi t^{2}} e^{-\left[\left(z_{M}-\xi\right)^{2}+\eta^{2}\right] / 4 t}
$$

while the eventually hitting probability to this point, the time integral of (8) , is

$$
E\left(z_{M}\right)=\frac{1}{\pi} \frac{|\eta|}{\left(z_{M}-\xi\right)^{2}+\eta^{2}}
$$

where the absolute value sign accounts for the possibility that the mosquito could initially be on either side of the man.

To appreciate the meaning of these results, we need to transform the above expressions for $E$ and $F$ to the original coordinates. For the eventual hitting probability $E$, the peak in this quantity occurs when $z_{M}=\xi$. From Fig. 2, this condition translates to

$$
x_{M}=\sqrt{D_{M}} \cos \theta z_{M}=\sqrt{D_{M}} \cos \theta\left[\frac{x_{M}(0)}{\sqrt{D_{M}}} \cos \theta+\frac{x_{M}(0)+a}{\sqrt{D_{m}}} \sin \theta\right],
$$

which we recast, after some simple algebra, as

$$
x \equiv x_{M}-x_{M}(0)=a \sin ^{2} \theta=a \frac{D_{M}}{D_{M}+D_{m}} .
$$


As expected, if the mosquito diffuses quickly, the man will have barely moved when he is bitten. Conversely, if the mosquito diffuses slowly, the man will be close to the initial position of the mosquito when the bite occurs.

We now use $E(z) d z=E(x) d x$ to transform (9) to the original $x_{M}, x_{m}$ coordinates to give:

$$
E(x)=\frac{1}{\pi} \frac{|a| \sin \theta \cos \theta}{\left(x-a \sin ^{2} \theta\right)^{2}+(a \sin \theta \cos \theta)^{2}}
$$

for the probability that the man has moved a distance $x$ when he is first bitten by the mosquito. Using $\tan \theta=\sqrt{D_{M} / D_{m}}$, we can equivalently rewrite $E(x)$ in terms of the diffusivities only:

$$
E(x)=\frac{|a|}{\pi} \frac{\sqrt{D_{M} D_{m}}}{\left(D_{M}+D_{m}\right)}\left\{\left[x-a \frac{D_{M}}{D_{M}+D_{m}}\right]^{2}+\left[a \frac{\sqrt{D_{M} D_{m}}}{\left(D_{M}+D_{m}\right)}\right]^{2}\right\}^{-1},
$$

This expression holds for $a>0$ and $a<0$, and we should average over these two cases to account for the possibilities that the mosquito is initially to the right or to the left of the man. Thus the probability distribution of displacements after a single encounter with the mosquito again has the long-range Cauchy form first given in (3). Similarly, Eq. (8) can be transformed back to the original variables, to give the probability $F(x, t)$ that the man has moved a distance $x$ when he meets the mosquito at time $t$ :

$$
F(x, t)=\frac{|a|}{4 \pi t^{2} \sqrt{D_{M} D_{m}}} e^{-\frac{1}{4 t}\left\{\left(x \sqrt{\frac{D_{M}+D_{m}}{D_{M} D_{m}}}-a \sqrt{\frac{D_{M}}{D_{m}\left(D_{M}+D_{m}\right)}}\right)^{2}+\left(\frac{a}{\sqrt{D_{M}+D_{m}}}\right)^{2}\right\}} .
$$

Integrating this last expression over all positions, the distribution of times when the man first encounters the mosquito is

$$
f(t)=\frac{|a|}{\sqrt{4 \pi\left(D_{M}+D_{m}\right) t^{3}}} e^{-a^{2} /\left[4\left(D_{M}+D_{m}\right) t\right]} .
$$

This result is well known and is easily obtained from Eq. (44) because if one is concerned

only with the meeting time, one only needs the separation between the man and mosquito, and this variable diffuses with diffusion coefficient $D_{M}+D_{m}$.

\section{Multiple Encounters}

We now compute the probability that the man has moved a distance $x$ at the moment when he dies, which occurs when the man has been bitten $\mathcal{N}$ times. As a preliminary, the Fourier transform of the Cauchy step-length distribution (12) at the $n^{\text {th }}$ encounter with the mosquito (with $n \leq \mathcal{N}$ ) is:

$$
E_{n}(k) \equiv \int E_{n}(x) e^{i k x} d x=\frac{1}{\pi} \int \frac{B_{n-1}}{\left(x-A_{n-1}\right)^{2}+B_{n-1}^{2}} e^{i k x} d x=e^{i k A_{n-1}-|k| B_{n-1}}
$$


Here

$$
A_{n}=a \epsilon_{n} \frac{D_{M}(n)}{D_{M}(n)+D_{m}(n)}, \quad \quad B_{n}=a \frac{\sqrt{D_{M}(n) D_{m}(n)}}{D_{M}(n)+D_{m}(n)},
$$

as given in $(12 \mathrm{~b})$. Here $\epsilon_{n}= \pm 1$ is a random variable that assumes the values +1 or -1 , respectively, if the mosquito is displaced by $+a$ or $-a$ after the $n^{\text {th }}$ meeting with the man; these two events occur with probability $1 / 2$. Additionally, all relevant variables are now indexed by $n$, the number of man-mosquito encounters. For a given realization of $\epsilon_{0}, \epsilon_{1}, \cdots, \epsilon_{\mathcal{N}}$, the probability distribution of displacements of the man at the $\mathcal{N}^{\text {th }}$ encounter with the mosquito equals the product of the Fourier transforms of single-encounter displacement distributions:

$$
\mathcal{E}_{\mathcal{N}}(k) \equiv \prod_{n=1}^{\mathcal{N}} E_{n}(k)=\exp \left[\sum_{n=0}^{\mathcal{N}-1}\left(i k A_{n}-|k| B_{n}\right)\right]
$$

Inverting this expression (see Eq. (15)), the probability distribution at the moment when the man and mosquito have met $\mathcal{N}$ times, and for a given realization $\epsilon_{0}, \epsilon_{1}, \cdots, \epsilon_{\mathcal{N}}$, is

$$
\mathcal{E}_{\mathcal{N}}(x)=\frac{1}{\pi} \frac{\sum_{n} B_{n}}{\left(x-\sum_{n=0}^{\mathcal{N}-1} A_{n}\right)^{2}+\left(\sum_{n=0}^{\mathcal{N}-1} B_{n}\right)^{2}} .
$$

For the specific case where initially $D_{M}(0)=D_{m}(0)=1$ and the diffusivity of the man decreases by $1 / \mathcal{N}$ each time the mosquito bites the man, then $D_{M}(n)=\left(1-\frac{n}{\mathcal{N}}\right)$ and the man dies after $\mathcal{N}$ bites. For this choice

$$
A_{n}=a \epsilon_{n} \frac{1-n / \mathcal{N}}{2-n / \mathcal{N}} \equiv a \epsilon_{n} f(n / \mathcal{N}), \quad B_{n}=|a| \frac{\sqrt{1-n / \mathcal{N}}}{2-n / \mathcal{N}} \equiv|a| g(n / \mathcal{N}) .
$$

To estimate the average of the probability distribution (17) over all realizations of $\epsilon_{0}, \epsilon_{1}, \epsilon_{2}, \cdots, \epsilon_{\mathcal{N}}$, we need $\sum_{n} A_{n}$ and $\sum_{n} B_{n}$. Because $f(z)$ and $g(z)$ are slowly varying functions of $z$, the leading behaviors of these sums are $\sum_{n} A_{n} \simeq \mathcal{N}^{1 / 2} a \alpha$, and $\sum_{n} B_{n} \simeq \mathcal{N} a \beta$, where $\alpha, \beta$ are of the order of one. Thus the probability distribution of displacements of the man when he dies is again the Cauchy distribution

$$
\mathcal{E}_{\mathcal{N}}(x)=\frac{1}{\pi} \frac{\mathcal{N}|a| \beta}{\left(x-\mathcal{N}^{1 / 2} a \alpha\right)^{2}+(\mathcal{N} a \beta)^{2}} \sim \frac{1}{\pi} \frac{\mathcal{N}|a| \beta}{x^{2}+(\mathcal{N} a \beta)^{2}} .
$$

Thus, while the typical distance that the man moves before he dies is of the order of $\mathcal{N}$, the long tail of this distribution leads to the average distance traveled being infinite. In this result, the displacement of the mosquito by $\pm a$ after each bite contributes only to the subdominant term $\mathcal{N}^{1 / 2} a \alpha$ in $\mathcal{E}_{\mathcal{N}}(x)$ and therefore is asymptotically irrelevant.

Following this same approach, we may also compute the probability $f_{\mathcal{N}}(t)$ that the man dies at time $t$ (which occurs after $\mathcal{N}$ mosquito bites). This probability is the convolution of the single-encounter first-passage probabilities (14). For this convolution, we first need the Laplace transform of Eq. (14). This is given by

$$
\begin{aligned}
f(s)=\int_{0}^{\infty} f(t) e^{-s t} d t & =\int_{0}^{\infty} \frac{|a|}{\sqrt{4 \pi\left(D_{M}+D_{m}\right) t^{3}}} e^{-a^{2} /\left[4\left(D_{M}+D_{m}\right) t\right]} e^{-s t} d t \\
& =e^{-|a| \sqrt{s /\left(D_{M}+D_{m}\right)}} .
\end{aligned}
$$


For $\mathcal{N}$ encounters with the mosquito, the Laplace transform is given by

$$
f_{\mathcal{N}}(s)=\prod_{n=0}^{\mathcal{N}-1} e^{-|a| \sqrt{s /(2-n / \mathcal{N})}}
$$

where we have used $D_{m}=1$ and $D_{M}=\left(1-\frac{n}{\mathcal{N}}\right)$. We now write the product as the exponential of the sum and replace the sum by an integral. The resulting integral is elementary and the result is

$$
f_{\mathcal{N}}(s)=e^{-w \sqrt{s}}
$$

where $w=2(\sqrt{2}-1)|a| \mathcal{N}$. Inverting this Laplace transform gives the distribution of times when the man dies, which happens after $\mathcal{N}$ encounters with the mosquito:

$$
f_{\mathcal{N}}(t)=\frac{w}{\sqrt{4 \pi t^{3}}} e^{-w^{2} / 4 t}
$$

For large times, this first-passage probability at the moment of death decays as $\mathcal{N} / t^{3 / 2}$. The mean time to die is infinite, as is the case for a single man-mosquito encounter. However, as $\mathcal{N}$ increases, corresponding to each mosquito bite being less damaging, the amplitude of the asymptotic distribution increases linearly with $\mathcal{N}$.

\section{Discussion}

We introduced a diffusive capture process in which each interaction between a prey and a predator causes the diffusivity of the prey to decrease by a fixed amount. After a finite number of interactions of this type, the prey eventually comes to rest, which we define as its death. As in the conventionally-studied case where the predator kills the prey when they first meet, basic questions about this capture process are the survival probability of the prey and the properties of its motion during the chase.

In this work, we investigated the simple case of a single predator, for which the above long-time properties of the prey can be obtained from classic first-passage concepts. Specifically, we determined the distribution of displacements when the prey dies. Between each encounter with the mosquito, the displacement distribution is given by the long-ranged Cauchy distribution. Thus the displacement distribution when the man dies - which is a convolution of single-encounter distributions - also has a Cauchy form that is given by Eq. (18). Thus even though the prey is continuously hunted by the predator and its health - quantified by its diffusivity - is progressively declining, the prey can, on average, endure to wander over a wide range.

There are a number of natural directions for further work. The most immediate is the case of $N \geq 2$ mosquitoes, where the man has to escape a cloud of mosquitoes rather than just a single mosquito. The special case of $N=2$ mosquitoes should be amenable to an asymptotic solution because the dynamics of this three-particle system after each mosquito bite is, in principle, known. When the mosquitoes and the man initially have the same diffusivities, the probability that the first bite occurs at time $t$ 
or greater decays as $t^{-3 / 4}$, for the initial ordering $M m m$ ( $M=$ man, $m=$ mosquito), and as $t^{-3 / 2}$, for the initial ordering $m M m$. (Note that the relative ordering of the man and the mosquito is important only in one dimension.) After many mosquito bites, the corresponding exponents for these same orderings are [15]:

$$
\begin{aligned}
& \beta_{\mathrm{Mmm}}=\left[2-\frac{2}{\pi} \cos ^{-1}\left(\frac{D_{M}}{1+D_{M}}\right)\right]^{-1} \sim 1-\frac{D_{M}}{\pi} \quad D_{M} \rightarrow 0, \\
& \beta_{\mathrm{mMm}}=\pi\left[2 \cos ^{-1}\left(\frac{D_{M}}{1+D_{M}}\right)\right]^{-1} \quad \sim 1+D_{M} \quad D_{M} \rightarrow 0 .
\end{aligned}
$$

Thus as the man is repeatedly bitten and becomes more sluggish, his survival probability decays with an exponent that approaches one as $D_{M} \rightarrow 0$, independent of the relative positions of the man and the mosquitoes. The effect of this exponent approaching 1 is that that the average lifetime of the man is infinite, even if he is initially surrounded by the mosquitoes, a configuration that would lead to a finite lifetime when a single mosquito bite is fatal.

For larger $N$, the basic question is to determine the dependence of the lifetime of the man as a function of $N$ and the initial diffusivities of the man and the mosquitoes. In general, when a single mosquito bite is fatal, the mean lifetime of the man is finite (the only exceptions are the cases of $N=1$, as well as $N=2$ and $N=3$, with all the mosquitoes initially to one side of the man). It will be worthwhile to determine the dependence of the lifetime on $N$ and on the virulence of the mosquitoes, as quantified by $\Delta D$, the decrease in the diffusivity of the man after each bite. What is an open question is the nature of the dependence of the man's lifetime on basic parameters in two dimensions or greater.

The approach developed here can, in principle, be extended to compute the joint distribution that the man has traveled a distance $x$ and has lived for a time $t$ when he dies. Starting with the joint probability (13) that the man has traveled a distance $x$ over a time $t$ when an encounter occurs, the corresponding distribution after $\mathcal{N}$ encounters with the mosquito is the convolution of these single-encounter joint probabilities. The calculation of these convolutions is conceptually straightforward by making use of the Laplace-Fourier transform technique. However, the inversion of this transforms does not appear to be tractable.

We thank Raphael Voituriez for helpful discussions. We also thank NSF Grant No. DMR-1205797 (SR) and ERC starting Grant No. FPTOpt-277998 (OB) for partial support of this research. 
[1] M. Bramson and D. Griffeath, "Capture problems for coupled random walks," in Random Walks, Brownian Motion, and Interacting Particle Systems: A Festschrift in Honor of Frank Spitzer, R. Durrett and H. Kesten, eds. (Birkhäuser, Boston, 1991).

[2] H. Kesten, "An absorption problem for several Brownian motions", in Seminar on Stochastic Processes, 1991, E. Çinlar, K. L. Chung, and M. J. Sharpe, eds. (Birkhäuser, Boston, 1992).

[3] J. Baik, Commun. Pure Appl. Math. 53, 1385 (2000).

[4] W. V. Li and Q.-M. Shao, Probab. Theor. Rel. Fields 122, 494 (2002).

[5] M. Katori and H. Tanemura, Phys. Rev. E 66, 011105 (2002).

[6] M. E. Fisher, J. Stat. Phys. 34, 667 (1984).

[7] S. Redner and K. Kang, J. Phys. A 17, L451 (1984).

[8] A. Blumen, G. Zumofen, and J. Klafter, Phys. Rev. B 30, 5379 (1984).

[9] M. E. Fisher and M. P. Gelfand, J. Stat. Phys. 53, 175 (1988).

[10] A. J. Bray, S. N. Majumdar, and G. Schehr, Adv. Phys. 62, 225 (2013).

[11] P. L. Krapivsky and S. Redner, J. Phys. A 29, 5347 (1996).

[12] S. Redner and P. L. Krapivsky, Am. J. Phys. 67, 1277 (1999).

[13] W. Feller, An Introduction to Probability Theory (J. S. Wiley \& Sons, New York, 1971), Vol. 1.

[14] G. H. Weiss, Aspects and Applications of the Random Walk (North-Holland, Amsterdam, 1994).

[15] S. Redner, A Guide to First-Passage Processes (Cambridge University Press, 2001).

[16] H. S. Carslaw and J. C. Jaeger, Conduction of Heat in Solids (Oxford University Press, Oxford, U. K., 1959).

[17] D. ben-Avraham, B. M. Johnson, C. A. Monaco, P. L. Krapivsky, and S. Redner, J. Phys. A 36, 1789 (2003).

[18] L. Breiman, Proc. Fifth Berkeley Symp. Math. Statist. and Probab. 2, 9 (1966).

[19] K. Uchiyama, Z. Wahrsch. verw. Gebiete 54, 75 (1980).

[20] P. Salminen, Adv. Appl. Prob. 20, 411 (1988).

[21] L. Turban, J. Phys. A 25, L127 (1992).

[22] F. Iglói, Phys. Rev. A 45, 7024 (1992); F. Iglói, I. Peschel, and L. Turban, Adv. Phys. 42, 683 (1993).

[23] P. L. Krapivsky and S. Redner, Am. J. Phys. 64, 546 (1996).

[24] O. Bénichou, N. Meunier, S. Redner, and R. Voituriez, Phys. Rev. E 85, 021137 (2012).

[25] D. J. Grabiner, Ann. Inst. H. Poincaré Prob. Stat. 35, 177 (1999). 